\title{
Dyslipidemia in AIDS patients on highly active antiretroviral therapy
}

\begin{abstract}
Highly active antiretroviral therapy (HAART) reduces AIDS-related morbidity and mortality, however it has been associated with metabolic abnormalities. This study estimated the prevalence of lipid abnormalities and related factors among patients on HAART. A cross-sectional study was conducted on adult patients, in central Brazil. Patients were interviewed, and blood obtained for lipids measurement. Dyslipidemia was defined as total cholesterol (TC) $\geq 240 \mathrm{mg} / \mathrm{dL}$, low-density lipoprotein $(\mathrm{LDL}) \geq 160 \mathrm{mg} / \mathrm{dL}$, triglycerides (TG) $>200$ and/or high-density lipoprotein (HDL) $<40 \mathrm{mg} / \mathrm{dL}$. Multiple logistic regression analyses were performed (SPSS 13.0). One hundred and thirteen patients were recruited. Mean age was 39.3 years; $68.1 \%$ were males; $50.4 \%$ were on nucleoside reverse transcriptase inhibitors (NRTI) in combination with non-nucleoside reverse transcriptase inhibitors (NNRTI), while $42.5 \%$ were on NRTI in combination with protease inhibitors (PIs). The prevalence of dyslipidemia was $66.7 \%$. Low HDL was the most frequent abnormality (53.5\%), followed by high TG (36.1\%). Patients on a PI regimen had a 5.2-fold higher risk (95\% CI: 1.8-14.8) of dyslipidemia, even after adjusting for sex, age, and duration of HIV infection/AIDS. The study discloses a high prevalence rate of dyslipidemia and points out a need for intervention programs to reduce future cardiovascular events in patients, on HAART.
\end{abstract}

Keywords: prevalence; dyslipidemia; HIV; AIDS; HAART.

[Braz J Infect Dis 2011;15(2):151-155]@Elsevier Editora Ltda.

\section{INTRODUCTION}

Highly active antiretroviral therapy (HAART) reduces the clinical manifestations of HIV infection, delaying the onset of AIDS and substantially improving the prognosis and quality of life of HIV-infected individuals. ${ }^{1,2}$

Since HAART was first introduced, the trend towards a reduced morbidity and mortality of HIV/AIDS patients has been accompanied by the emergence of side effects and toxicities in various organs. ${ }^{3}$ Metabolic abnormalities such as dyslipidemias, insulin resistance, hyperglycemia and changes in body fat distribution or lipodystrophy syndrome are classic examples of this situation. ${ }^{4}$ Side effects reduces adherence to treatment, and long term exposure to dyslipidemias in this population may increase the risk of cardiovascular diseases. ${ }^{5-7}$

Antiretroviral drugs have been widely available in Brazil, since 1996 and approximately 170,000 patients had access to HAART universally distributed, by the Brazilian National Health System (SUS). ${ }^{8}$ Analyses of the impact of
HAART in Brazil have shown a pattern similar to that described in developed countries, i.e. a significant reduction in opportunistic diseases with a consequent reduction in AIDS-related hospitalizations and mortality. ${ }^{9}$

Dyslipidemias are estimated to be frequent events in patients using HAART; nevertheless, up to the present time, few studies have investigated the prevalence of these abnormalities in Brazil. ${ }^{10-12}$ The present study aimed to estimate the prevalence of dyslipidemias in HIVinfected patients on antiretroviral therapy, in the central part of Brazil, and to evaluate factors associated with these abnormalities. These data may help to predict the impact of longterm antiretroviral therapy on cardiovascular diseases in this group.

\section{MATERIAL AND METHODS}

HIV-infected patients over 19 years of age, who had been in use of antiretroviral therapy for at least 30 days, were eligible to participate in this study. All of them were registered in the main re-
Authors

Max Weyler Nery ${ }^{1}$

Celina Maria Turchi Martelli ${ }^{2}$

Marília Dalva Turchi ${ }^{2}$

${ }^{1} \mathrm{MD}, \mathrm{MSc}$; Departamento de Medicina da Pontifícia Universidade Católica de Goiás, Brazil

${ }^{2} \mathrm{MD}, \mathrm{PhD}$; Instituto de Patologia Tropical e Saúde Pública da Universidade Federal de Goiás, Brazil

Submitted on: 03/05/2010 Approved on: 10/30/2010

Correspondence to:

Max Weyler Nery

Departamento de

Medicina da Pontifícia

Universidade Católica de Goiás

Av. Universitária, 1440, Setor Universitário, Área IV, CEP: 74.605-010

Goiânia, Goiás, Brasil

Phone: (62) 32504000 , 32504014

Fax: (62) 32504024 maxwnery@uol.com.br

We declare no conflict of interest. 
ferral center for HIV/AIDS patients, Hospital for Tropical Diseases Dr. Anuar Auad (HDT/AA), in the city of Goiânia, Goiás.

This is a prevalence study with convenience sampling. Sample size was calculated to detect prevalences of dyslipidemia $\geq 40 \%$ in patients on antiretroviral therapy with $10 \%$ precision, resulting in a required sample of 93 patients. Cases were identified between 2005 and 2006.

The protocol was approved by the Internal Review Board of the Universidade Federal de Goiás. After obtaining informed consent, patients were interviewed, their medical and laboratory records abstracted, and blood obtained for laboratory testing. Patients with a history of opportunistic disease diagnosed less than two months prior to the interview were excluded from the study.

A structured interview was administered to all participants by the principal investigator to collect social, demographic and clinical data. Patient's clinical charts were also used as data sources from which information on duration of antiretroviral therapy, T-CD4+ lymphocyte count, HIV-1 viral load and previous lipid evaluations was obtained. For the purposes of analyses, viral load measurement was dichotomized into detectable or undetectable, and T-CD4+ lymphocyte count was classified as $>350$ or $\leq 350$ cells $/ \mathrm{mL}$. When more than one laboratory evaluation was available, the most recent one was used.

Following the interview, the patients were referred for blood lipid evaluation, performed after an overnight fasting period. Lipid profile was obtained in individuals who were metabolically stable ${ }^{13}$ by measuring total cholesterol (TC), high-density lipoprotein cholesterol (HDL-C) and triglycerides (TG) using an automated enzymatic method. Low-density lipoprotein cholesterol (LDL-C) was calculated using Friedwald's formula in individuals with triglycerides $\leq 400$ $\mathrm{mg} / \mathrm{dL}$. Blood lipids were classified as categorical variables and the following situations were defined as dyslipidemia: $\mathrm{TC} \geq 240 \mathrm{mg} / \mathrm{dL}, \mathrm{LDL}-\mathrm{C} \geq 160 \mathrm{mg} / \mathrm{dl}, \mathrm{TG}>200 \mathrm{mg} / \mathrm{dL}$ and/ or HDL-C $<40 \mathrm{mg} / \mathrm{dL} \cdot{ }^{13}$ To assess cardiovascular risk, Castelli's Index I was calculated using the ratio: TC/HDL-C; a Castelli Index I $>5.1$ for men and $>4.4$ for women were considered indicative of an elevated risk. ${ }^{14}$

Descriptive and exploratory statistical methods were used: measurements of central tendency and dispersion for continuous variables, frequency distributions with their respective confidence intervals for categorical variables. Chi-square test or Fisher's exact test were used to evaluate differences in frequency distribution. Student's t-test was employed to evaluate differences between two means, and the Kruskal-Wallis nonparametric test to evaluate differences in duration of antiretroviral therapy. Prevalence rates of dyslipidemia were calculated with their respective $95 \%$ confidence intervals (95\% CI).

A case-control analysis was carried out to evaluate the association between dyslipidemia and exposure variables. Patients with dyslipidemia were considered "cases" and patients who did not have this condition were considered "con- trols". Odds ratios (OR) and their respective 95\% confidence intervals (95\% CI) were calculated. Multiple logistic regression models were applied with simultaneous adjustment for sex, age and length of time since HIV/diagnosis. Interaction between exposure variables were also tested to build-up the final logistic model. The correlation between the time on protease inhibitors and serum HDL levels was evaluated. A p-value of less than 0.05 was considered significant. Statistical analyses were performed using Statistical Package for Social Science software (version 13.0).

\section{RESULTS}

One hundred and thirteen HIV-infected patients on antiretroviral therapy were enrolled. The mean age of participants was $39.3 \pm 10.4$ years, ranging from 20 to 64 years; $68.1 \%$ were males; $52.2 \%$ had less than 9 years of schooling; and approximately half of them reported very low monthly income ( $\leq$ one minimum wage). T-CD4+ lymphocyte count $>350$ cells $/ \mathrm{mL}$ was found in $42.4 \%$ of patients, whereas viral load was undetectable in $51.9 \%$ of participants.

The most frequently prescribed antiretroviral regimen consisted of the combination of two nucleoside reverse transcriptase inhibitors (NRTI) with non-nucleoside reverse transcriptase inhibitors (NNRTI), used by $50.4 \%$ of the sample, followed by the combination of two NRTI with protease inhibitors (PI), used by $42.5 \%$ of study participants. Use of three distinct classes of antiretroviral drugs (NRTI, NNRTI and PI) was recorded for $4.4 \%$ of patients. The majority of patients (64.6\%) were on three drugs; $25.7 \%$ on four drugs and $8.0 \%$ on five or more drugs. The most commonly prescribed drugs were lamivudine (92.9\%), zidovudine (66.4\%), and efavirenz (49.6\%). Fifty-five patients (48.7\%) were on at least one PI.

Boosted ritonavir was used with other PI, in 37 patients. The most common combination was ritonavir with atazanavir, reported by 26 out of 37 patients (62.2\%).

Change in body fat distribution, after initiating antiretroviral therapy, was perceived by $39.8 \%$ of patients, with abdominal fat accumulation being the most frequently mentioned (91.1\%). Less than half the patients (45.1\%) reported having previous lipid assessment, and $47.0 \%$ of these patients reported increased cholesterol and/or triglyceride levels. Among those who reported abnormal blood lipid levels, $20.8 \%$ were on lipid-lowering drugs.

Out of 113 patients interviewed, 97 had were on antiretroviral therapy for at least 30 days and were evaluated with respect to their lipid profile. Length of time on PI was significantly shorter than the time on either NRTI or NNRTI ( $<<0.043)$.

Table 1 shows the results of the Castelli Index I according to sex and the lipid profile of 97 patients evaluated in accordance with the Brazilian Cardiology Society (SBC) classification. Among men 36.9\% had a Castelli Index I > 5.1, whereas $40 \%$ of the women had a Castelli Index I $>4.4$, indicating a higher risk of cardiovascular disease in this population. 
Table 1. Lipid profile of patients using antiretroviral therapy

\begin{tabular}{|c|c|c|c|}
\hline Variable $(\mathrm{mg} / \mathrm{dL}) \quad \mathrm{F}$ & Frequency & Percentage & $95 \%$ CI \\
\hline \multicolumn{4}{|l|}{ Total cholesterol } \\
\hline Optimal (<200) & 70 & 72.2 & $62.1-80.8$ \\
\hline $\begin{array}{l}\text { Borderline high } \\
(200 \text { - 239) }\end{array}$ & 17 & 17.5 & $10.6-26.6$ \\
\hline $\operatorname{High}(\geq 240)$ & 10 & 10.3 & $5.0-18.1$ \\
\hline \multicolumn{4}{|l|}{ HDL cholesterol ${ }^{\mathrm{a}}$} \\
\hline Low $(<40)$ & 51 & 53.7 & $43.1-64.0$ \\
\hline Optimal (> 60) & 44 & 46.3 & $36.0-56.8$ \\
\hline \multicolumn{4}{|l|}{ LDL cholesterol $^{\mathrm{b}}$} \\
\hline Optimal $(<100)$ & 45 & 50.6 & $39.7-61.3$ \\
\hline $\begin{array}{l}\text { Desirable } \\
(100-129)\end{array}$ & 26 & 29.2 & $20.0-40.0$ \\
\hline $\begin{array}{l}\text { Borderline high } \\
(130-159)\end{array}$ & 13 & 14.6 & $8.0-23.7$ \\
\hline High (160-189) & 3 & 3.4 & $0.7-9.5$ \\
\hline Very high $(\geq 190)$ & 2 & 2.2 & $0.3-7.9$ \\
\hline \multicolumn{4}{|l|}{ Triglycerides } \\
\hline Optimal (< 150) & 51 & 52.6 & $42.2-62.8$ \\
\hline $\begin{array}{l}\text { Borderline high } \\
(150-200)\end{array}$ & 11 & 11.3 & $5.8-19.4$ \\
\hline $\begin{array}{l}\text { High } \\
(201-499)\end{array}$ & 32 & 33.0 & $23.8-43.3$ \\
\hline Very high $(\geq 500)$ & 3 & 3.1 & $0.6-8.8$ \\
\hline \multicolumn{4}{|l|}{ Castelli Index I I,c,d } \\
\hline Men > 5.1 & 24 & 36.9 & $25.5-49.8$ \\
\hline Women $>4.4$ & 12 & 40.0 & $23.2-59.3$ \\
\hline
\end{tabular}

HDL, high density lipoprotein; LDL, low density lipoprotein. aData missing for 2 patients.

${ }^{b}$ Data missing for 8 patients.

'Castelli Index I, total cholesterol/HDL cholesterol.

${ }^{\mathrm{d}} 65$ male and 30 female participants.
The majority of patients (66.7\%) had at least one laboratory abnormality compatible with a diagnosis of dyslipidemia. The most frequent abnormality was low HDL-C, levels below $40 \mathrm{mg} / \mathrm{dL}$ being found in $53.7 \%$ of participants. Additionally, $36.1 \%$ of the participants had triglyceride levels $>200 \mathrm{mg} / \mathrm{dL}$. HDL-C $<40 \mathrm{mg} / \mathrm{dL}$ and triglycerides $>200 \mathrm{mg} / \mathrm{dL}$ was the most common mixed dyslipidemia, being diagnosed in $27.4 \%$ of participants.

Age, duration of HIV/AIDS, sex, viral load measurement and immunological status evaluated by T-CD4+ cell were not significantly different between dyslipidemic and nondyslipidemic patients.

In univariate analysis patients on PI had 3.4-fold greater risk (95\% CI: 1.4-8.4) of dyslipidemia compared to patients on regimens that did not include this class of drugs. On the other hand, patients on NNRTI-containing regimens had lower risk of dyslipidemia ( $\mathrm{OR}=0.3$; 95\% CI: 0.1-0.6). Nevertheless, a significant interaction $(p<0.003)$ was found between these exposure variables (being on a PI-containing regimen or on an NNRTI-containing regimen) and the presence of dyslipidemia.

PI were independently associated with dyslipidemia even after simultaneous adjustment for potential confounding factors such as sex, age and duration of HIV infection $\left(\mathrm{OR}_{\text {adjusted }}=5.2 ; 95 \%\right.$ CI: 1.8-14.8) as shown in Table 2. There was no correlation between duration of PI use (in months) and serum HDL-C level $(r=-0.2 ; p=0.12)$.

\section{DISCUSSION}

The present study discloses a high prevalence of dyslipidemia in patients on antiretroviral therapy in a large city in central Brazil. These findings suggest a potential risk for the development of cardiovascular diseases in a significant proportion of HIV/AIDS patients in coming years. Therefore, the benefits obtained with the use of HAART over the past years may be reduced if measures to minimize the negative impact of cardiovascular disease in this population are not implemented.

Table 2. Multiple logistic regression analysis of factors associated with dyslipidemia

\begin{tabular}{lcccc}
\hline Variable & \% dyslipidemia & OR ( 95\%CI) & OR $_{\text {adjusted }}$ (95\% CI) & p \\
\hline Use of PI & 79.6 & $3.4(1.4-8.4)$ & $5.2(1.8-14.8)$ & $0.002^{*}$ \\
\hline Male & 71.2 & $1.9(0.8-4.6)$ & $2.7(1.0-7.6)$ & 0.053 \\
\hline Age (years) & - & - & $1.0(1.0-1.1)$ & $0.013^{*}$ \\
\hline Time of HIV (months) & - & - & $1.0(1.0-1.0)$ & 0.360
\end{tabular}

PI, protease inhibitors; HIV, human immunodeficiency virus.

Age and time of diagnosis of HIV/AIDS were included in the model as continuous variables.

OR adjusted for sex, age and time of diagnosis of HIV infection/AIDS.

* Statistically significant association. 
The majority of HIV-infected patients on HAART, in our setting, were found to have abnormal lipid profile with potential risk for cardiovascular disease in a near future. ${ }^{7}$

Patients using lipid-lowering therapy were not excluded from the present analysis, and, unexpectedly, all of them had some lipid abnormality compatible with the diagnosis of dyslipidemia, suggesting the need for better clinical control, in accordance with the recommendations guidelines. ${ }^{13,15}$

There are wide variations in the prevalence rate of dyslipidemia among HIV-infected patients on antiretroviral therapy. Comparison of results from different prevalence studies should be made with caution, taking into consideration differences in methodology, populations studied, antiretroviral regimens used by the patients, and the cut-off points established to define lipid abnormalities. For the purpose of clinical management, the current trend is to attempt to lower blood lipid levels; hence, the cut-off point for the definition of lipid abnormalities is lower. ${ }^{13}$ However, in the present study, it was decided to apply more conservative criteria with higher cut-off limits to define dyslipidemia, thereby guaranteeing greater specificity of results. The percentage of lipid abnormalities encountered in this study would have been even higher if lower cut-off points had been used.

In our sample, low HDL was the most frequent abnormality ( $53.7 \%$ of patients had levels $<40 \mathrm{mg} / \mathrm{dL}$ ), followed by elevated triglyceride levels $(36.1 \%$ of patients had levels $>200 \mathrm{mg} / \mathrm{dL}$ ). Approximately $40 \%$ of patients had an elevated Castelli Index I, indicating an increased risk for atherosclerotic cardiovascular disease in this population. This index is considered a simple approach for lipid risk assessment, the high total cholesterol is a marker for atherogenic lipoproteins and low HDL cholesterol correlates with risk factors of metabolic syndrome. . $^{14,16}$

Few studies on the prevalence of dyslipidemia carried out in Brazil have been published. ${ }^{10-12}$ In the northeast region, a cross-sectional study ${ }^{12}$ conducted among a sample of 372 patients with and without antiretroviral therapy found high TC, low HDL and high TG levels in $20.1 \%, 57.1 \%$ and $26.5 \%$ of subjects, respectively. A study conducted in the southeas $\mathrm{t}^{11}$ involving 238 patients, predominantly using PI, found low HDL in 65\% of participants and hypertriglyceridemia in $46 \%$ of them, using different cut-off points from those used in our study.

Different antiretroviral drugs may be associated with abnormalities in lipid profile. Various studies have shown an association between the use of PI and dyslipidemia. ${ }^{5,7,12}$ The prevalence and degree of lipid abnormalities, however, vary between the different drugs, within a single class and possibly with duration of treatment. ${ }^{17}$ The present study was not designed to evaluate differences between drugs within the same class. The use of PI was associated with the presence of dyslipidemia even after simultaneous adjustment for possible confounding factors such as sex, age and time since diagnosis of HIV/AIDS. The duration of PI use was not included in the logistic regression model. Nevertheless, there was no evidence of any correlation between duration of PI use and serum HDL-C levels $(p=0.12)$. Contrary to our findings, another study conducted in Brazi ${ }^{11}$ reported a correlation between duration of PI use and HDL-C levels.

The association between NNRTI use and dyslipidemias is controversial. A growing number of reports suggest that use of this class of drugs may lead to an increase in lipids. ${ }^{17,18}$ In agreement with our results, another study conducted in London ${ }^{19}$ found no increase in total cholesterol or in triglycerides in patients on NNRTI. Other investigators have reported an increase in HDL-C in patients on this class of drugs, which may have a protective effect against the development of coronary disease. ${ }^{20}$

One of the limitations of the present study is not having included the cumulative history of the prior use of antiretrovirals. It is possible that patients on PI, mainly those on ritonavir-boosted regimens, may have had the disease for a longer time and may have been exposed to various drugs previously, which may have interfered in their current lipid profile. To minimize this effect, an estimation of the time of HIV infection/AIDS was included in the logistic regression model as surrogate marker for the time of use of antiretroviral drugs.

Some other methodological issues with respect to the site at which cases were recruited and the sampling process should also be mentioned. Patients registered at the principal referral service for HIV/AIDS in the state of Goiás were recruited to this study. This referral center is responsible for the notification of almost all cases of AIDS in the state.

Despite $48.1 \%$ of these patients had a detectable viral load we cannot conclude that they were inappropriately treated. Viral load assessment is used to monitor therapy and around half these patients underwent a change in their antiretroviral regimen following evaluation of plasma viremia.

Assuming that the sample of patients studied is representative, our results indicate a high prevalence of dyslipidemia and risk for the development of cardiovascular disease in a significant proportion of patients with HIV/AIDS in the years to come in central part of Brazil. Furthermore, as most of patients unaware of their lipid abnormalities, the findings of this study underscore the need to adopt strategies to routinely evaluate and modify the risk of cardiovascular diseases in this population.

\section{ACKNOWLEDGEMENTS}

The authors are grateful to the directors of the Hospital for Tropical Diseases Dr. Anuar Auad (HDT/AA) of Goiás State Department of Health. 


\section{REFERENCES}

1. Palella FJ, Jr., Delaney KM, Moorman AC et al. Declining morbidity and mortality among patients with advanced human immunodeficiency virus infection. HIV Outpatient Study Investigators. N Engl J Med. 1998 Mar 26; 338(13):853-60.

2. Enanoria WT, Ng C, Saha SR, Colford Jr JM. Treatment outcomes after highly active antiretroviral therapy: a meta-analysis of randomised controlled trials. Lancet Infect Dis. 2004; 4(7):414-25.

3. Carr A, Cooper DA. Adverse effects of antiretroviral therapy. Lancet. 2000; 356(9239):1423-30.

4. Wohl DA, McComsey G, Tebas P et al. Current concepts in the diagnosis and management of metabolic complications of HIV infection and its therapy. Clin Infect Dis. 2006; 43(5):645-53.

5. Rhew DC, Bernal M, Aguilar D, Iloeje U, Goetz MB. Association between protease inhibitor use and increased cardiovascular risk in patients infected with human immunodeficiency virus: a systematic review. Clin Infect Dis. 2003; 37(7):959-72.

6. Saves M, Chene G, Ducimetiere P et al. Risk factors for coronary heart disease in patients treated for human immunodeficiency virus infection compared with the general population. Clin Infect Dis., 2003; 37(2):292-8.

7. Friis-Moller N, Reiss P, Sabin CA et al. Class of antiretroviral drugs and the risk of myocardial infarction. N Engl J Med. 2007; 356(17):1723-35.

8. Grangeiro A Teixeira L, Bastos FI, Teixeira P. Sustainability of Brazilian policy for access to antiretroviral drugs. Rev Saude Publica. 2006; 40 Suppl:60-9.

9. Dourado I, Veras MA, Barreira D, de Brito AM. AIDS epidemic trends after the introduction of antiretroviral therapy in Brazil. Rev Saude Publica. 2006; 40 Suppl:9-17.

10. Caramelli B, de Bernoche CY, Sartori AM et al. Hyperlipidemia related to the use of HIV-protease inhibitors: natural history and results of treatment with fenofibrate. Braz J Infect Dis. 2001; 5(6):332-8.

11. Albuquerque EM, de Faria EC, Oliveira HC, Magro DO, Castilho LN. High frequency of Fredricksons phenotypes IV and IIb in Brazilians infected by human immunodeficiency virus. BMC Infect Dis. 2005; 5(1):47.
12. de Araujo PS, de Alencar Ximenes RA, Lopes CF, Duarte JY, da Silva MM, Carneiro EM. Antiretroviral treatment for HIV infection/AIDS and the risk of developing hyperglycemia and hyperlipidemia. Rev Inst Med Trop São Paulo. 2007; 49(2):73-8.

13. SBC. III Brazilian Guidelines on Dyslipidemias and Guideline of Atherosclerosis Prevention from Atherosclerosis Department of Sociedade Brasileira de Cardiologia. Arq Bras Cardiol. 2001;77 Suppl 3:1-48.

14. Castelli WP, Abbott RD, McNamara PM. Summary estimates of cholesterol used to predict coronary heart disease. Circulation. 1983; 67(4):730-4.

15. Dube MP, Stein JH, Aberg JA et al. Guidelines for the evaluation and management of dyslipidemia in human immunodeficiency virus (HIV)-infected adults receiving antiretroviral therapy: recommendations of the HIV Medical Association of the Infectious Disease Society of America and the Adult AIDS Clinical Trials Group. Clin Infect Dis. 2003; 37(5):613-27.

16. Wilson PW, D’Agostino RB, Levy D, Belanger AM, Silbershatz H, Kannel WB. Prediction of coronary heart disease using risk factor categories. Circulation. 1998; 97(18):1837-47.

17. Fontas E, van Leth F, Sabin CA et al. Lipid profiles in HIVinfected patients receiving combination antiretroviral therapy: are different antiretroviral drugs associated with different lipid profiles? J Infect Dis. 2004; 189(6):1056-74.

18. Friis-Moller N, Weber R, Reiss $\mathrm{P}$ et al. Cardiovascular disease risk factors in HIV patient - association with antiretroviral therapy. Results from the DAD study. Aids. 2003; 17(8):117993.

19. Matthews GV, Moyle GJ, Mandalia S, Bower M, Nelson M, Gazzard BG. Absence of association between individual thymidine analogues or nonnucleoside analogues and lipid abnormalities in HIV-1-infected persons on initial therapy. J Acquir Immune Defic Syndr. 2000; 24(4):310-5.

20. van der Valk M, Kastelein JJ, Murphy RL et al. Nevirapinecontaining antiretroviral therapy in HIV-1 infected patients results in an anti-atherogenic lipid profile. Aids. 2001; 15(18):2407-14. 